TRANSACTIONS OF THE

AMERICAN MATHEMATICAL SOCIETY

Volume 348, Number 6, June 1996

\title{
BOTT'S VANISHING THEOREM FOR REGULAR LIE ALGEBROIDS
}

\author{
JAN KUBARSKI
}

\begin{abstract}
Differential geometry has discovered many objects which determine Lie algebroids playing a role analogous to that of Lie algebras for Lie groups. For example:

- differential groupoids,

- principal bundles,

— vector bundles,

- actions of Lie groups on manifolds,

- transversally complete foliations,

- nonclosed Lie subgroups,

- Poisson manifolds,

- some complete closed pseudogroups.

We carry over the idea of Bott's Vanishing Theorem to regular Lie algebroids (using the Chern-Weil homomorphism of transitive Lie algebroids investigated by the author) and, next, apply it to new situations which are not described by the classical version, for example, to the theory of transversally complete foliations and nonclosed Lie subgroups in order to obtain some topological obstructions for the existence of involutive distributions and Lie subalgebras of some types (respectively).
\end{abstract}

\section{INTRODUCTION}

In [K3] the author defined the Chern-Weil homomorphism

$$
h_{A}: I^{\circ}(A) \rightarrow H_{F}(M)
$$

of a regular Lie algebroid $(A, \llbracket \cdot, \cdot \rrbracket, \gamma)$ over a foliated manifold $(M, F)$, generalizing the well-known notion from the theory of principal bundles. (Every principal bundle has a transitive Lie algebroid [K1], [MA] - more precisely, there are three different but equivalent such constructions.) In (1.1) $F=\operatorname{Im} \gamma \subset T M$, and $H_{F}(M)$ is the algebra of tangential cohomology classes. Besides, $\boldsymbol{g}=$ Ker $\gamma$ is the adjoint Lie algebra bundle of $A$ and $I^{\circ}(A):=\bigoplus^{k \geq 0} I^{\circ k}(A)$ where $I^{\circ k}(A):=\left(\operatorname{Sec}\left(\bigvee^{k} \boldsymbol{g}^{\star}\right)\right)_{I^{\circ}(A)}$ is the space of cross-sections of the symmetric $k$-power of $\boldsymbol{g}^{\star}$ which are invariant with respect to the "adjoint representation" of $A$ on $\boldsymbol{g}$. We recall that $\Gamma \in I^{\circ k}(A)$

Received by the editors May 27, 1994.

1991 Mathematics Subject Classification. Primary 22E15, 22E60, 53C05, 57T10, 57R20.

Key words and phrases. Bott's phenomenon, Chern-Weil homomorphism, Lie algebroid, transversally complete foliation, nonclosed Lie subgroup.

(C)1996 American Mathematical Society 
if and only if

$$
\begin{aligned}
\forall_{\xi \in \operatorname{Sec} A} \forall_{\sigma_{1}, \ldots, \sigma_{k} \in \operatorname{Sec} \boldsymbol{g}}( & (\gamma \circ \xi)\left\langle\Gamma, \sigma_{1} \vee \ldots \vee \sigma_{k}\right\rangle \\
& \left.=\sum_{i=1}^{k}\left\langle\Gamma, \sigma_{1} \vee \ldots \vee \llbracket \xi, \sigma_{i} \rrbracket \vee \ldots \vee \sigma_{k}\right\rangle\right) .
\end{aligned}
$$

The construction of the Chern-Weil homomorphism of regular Lie algebroids is the above-mentioned generalization due to the following:

Theorem 1.1. [K3, Th.5.6.1] For a principal bundle $P=P(M, G)$, there exists a natural monomorhism of algebras $\nu:\left(\bigvee^{\star}\right)_{I(G)} \longrightarrow I^{\circ}(A(P))$ such that $h_{A(P)} \circ$ $\nu=h_{P}\left(\mathfrak{g}\right.$ denotes the Lie algebra of $G,\left(\bigvee \mathfrak{g}^{\star}\right)_{I(G)}$ - the space of $G$-invariant polynomials and $h_{P}$ - the Chern-Weil homomorphism of $P$ ). $\nu$ is an isomorphism if $P$ is connected ( $G$ may be disconnected !).

This theorem also means that the Chern-Weil homomorphism of a principal bundle $P$ is a characteristic feature of its Lie algebroid $A(P)$ (provided only that $P$ is connected). We pay our attention to the fact that this holds although in the Lie algebroid $A(P)$ there is no direct information about the structure Lie group of $P$ (which may be disconnected)!

Remark 1.2. It should be added that many years ago N. Teleman in 1972 [T1], [T2] had constructed the Chern-Weil characteristic classes for some exact sequences of $R$-Lie algebras and $\mathcal{F}$-modules $0 \rightarrow H \rightarrow P \rightarrow T \rightarrow 0(R=$ commutative ring with $1, \mathcal{F}=$ commutative $R$-algebra with 1$)$. For the case of the exact sequence of real Lie algebras and $\Omega^{\circ}(M)$-modules $\quad 0 \rightarrow \operatorname{Sec} \boldsymbol{g} \rightarrow \operatorname{Sec} A \rightarrow \operatorname{Sec} F \rightarrow 0$ of the cross-sections corresponding to the Atiyah sequence of a regular Lie algebroid $A$ over a foliated manifold $(M, F)$, one obtains in this way the characteristic classes constructed in [K3] via (1.1). Teleman had also noticed that his construction is a generalization of the Chern-Weil characteristic classes of principal bundles provided that the structure Lie groups are connected. The same result was repeated in [K1] by the method of keeping the apparatus of principal bundles only. The final solution of the mutual relation between the Chern-Weil characteristic classes of principal bundles and of their Lie algebroids is done in Th. 1.1 above. We add that to prove this fact, the theory of representations of principal bundles on vector bundles was initiated.

By using the fact (discovered by P. Molino [MO1]) that possess a transitive Lie algebroid, it is possible to apply the Chern-Weil homomorphism to these foliations. The significance of this homomorphism is as follows:

Theorem 1.3. [K3] The nontriviality of the Chern-Weil homomorphism of the Lie algebroid $A(M, \mathcal{F})$ of a transversally complete foliation $(M, \mathcal{F})$ means the nonexistence of an involutive distribution $C \subset T M$ such that (i) $C \cap E_{b}=E$, (ii) $C+E_{b}=T M$, (iii) $C_{\mid x}=\{X(x) ; X \in L(M, \mathcal{F}) \cap \operatorname{Sec} C\}$ for each $x \in M$, where $E$ and $E_{b}$ denote the vector bundles tangent to $\mathcal{F}$ and to the basic foliation $\mathcal{F}_{b}$, respectively. [We add that each involutive distribution $C$ satisfying conditions (i)-(iii) determines a flat connection in $A(M, \mathcal{F})$.] Equivalently, this means the nonexistence of a vector subbundle $\tilde{Q}$ of the transverse bundle $Q$, such that (i) $Q=Q^{\prime} \oplus \tilde{Q}$ $\left(Q^{\prime}=E_{b} / E\right)$, (ii) $\tilde{Q}_{\mid x}=\left\{\xi_{x} ; \xi \in l(M ; \mathcal{F}) \cap \operatorname{Sec} \tilde{Q}\right\}$ for each $x \in M$.

The answer to the question concerning the existence of transversally complete foliations whose Lie algebroids possess the nontrivial Chern-Weil homomorphism 
is: YES. The fact that, among them, there are ones whose Lie algebroids are not integrable (i.e. which are not isomorphic to the Lie algebroid of any principal bundle), see Th.1.4 below, takes a great significance in differential geometry. It indicates, for example, that the method of Lie algebroids in differential geometry acquires a great value beyond the theory of principal bundles (and differential groupoids).

Theorem 1.4. [K3] If $G$ is a connected, compact and semisimple Lie group and $H \subset G$ is its arbitrary connected nonclosed Lie subgroup, then the Chern-Weil homomorphism of the foliation of left cosets of $G$ by $H$ is nontrivial. Adding the simple connectedness of $G$, we get, according to Almeida-Molino's Theorem [A-M], a nonintegrable transitive Lie algebroid having the nontrivial Chern-Weil homomorphism.

This paper is devoted to carrying the idea of Bott's Vanishing Theorem over to regular Lie algebroids. Namely, we check

Theorem 1.5 (Bott's Vanishing Theorem). Let $(A, \llbracket \cdot, \cdot \rrbracket, \gamma)$ be a regular Lie algebroid over $(M, F)$ and $\operatorname{Pont}(A):=\operatorname{Im}\left(h_{A}\right) \subset H_{F}(M)$ the Pontryagin algebra of $A$. If $A$ admits a flat partial connection $\lambda^{\prime}$ over an involutive distribution $F^{\prime} \subset F \subset T M$, then

$$
\operatorname{Pont}^{p}(A)=0 \quad \text { for } \quad p \geq 2 \cdot(q+1)
$$

where $q=\operatorname{rank}\left(F / F^{\prime}\right)$. If, moreover, $\lambda^{\prime}$ admits a basic adapted connection, then

$$
\operatorname{Pont}^{p}(A)=0 \quad \text { for } \quad p \geq q+1 \text {. }
$$

This theorem is (in this paper) interpreted for transversally complete foliations and nonclosed Lie subgroups.

\section{IDEALS AND VECTOR BUNDLES}

Let $V, W$ be any real vector spaces. Recall that $[\mathrm{G}]$

- If $W \subset \wedge^{p} V$, then the left ideal $I_{W} \subset \wedge V$ generated by $W$ has a gradation, i.e. $I_{W}=\bigoplus^{h \geq 0} I_{W}^{(h)}$ where $I_{W}^{(h)}=I_{W} \cap \bigwedge^{h} V$; hence $I_{W}$ is two-sided. Of course, $I_{W}^{(h)}=0$ for $h<p$.

- If $W \subset V$, then the $p$-th power of $I_{W},\left(I_{W}\right)^{p}$, equals $I \bigwedge^{p} W$.

For $W \subset V$, we put $W^{\perp}=\left\{v^{\star} \in V^{\star} ; \forall w \in W,\left\langle v^{\star}, w\right\rangle=0\right\}$.

The following proposition entirely describes the ideal $I \bigwedge^{p}\left(W^{\perp}\right)$.

Proposition 2.1. (See, for example, $[\mathrm{A}]$ ). In the case of a finite-dimensional vector space $V$, we have, for $h \geq p, \phi \in I_{\bigwedge^{p}\left(W^{\perp}\right)}^{(h)} \Leftrightarrow \forall a \in \bigwedge^{h-p+1} W, i(a)(\phi)=0$ (for the operator $i(a)$, see $[\mathrm{G}]$ ).

The above theorem remains valid also in the infinite-dimensional case [J. Kubarski, unpublished].

Take now two vector bundles $F^{\prime}$ and $F$ on a paracompact manifold $M$, such that $F^{\prime} \subset F$, and define, for $p \geq 1$, a vector subbundle $I \bigwedge^{p} F^{\prime}$ of $\bigwedge F$ by

$$
I_{\bigwedge^{p} F^{\prime}}:=\bigcup_{x \in M} I_{\bigwedge^{p}\left(F_{\mid x}^{\prime}\right)} \subset \bigwedge F
$$

Of course, the space of global $C^{\infty}$-cross-sections $\operatorname{Sec}\left(I \bigwedge^{p} F^{\prime}\right)$ sets up an ideal in the algebra $\operatorname{Sec}(\bigwedge F)$. Using the paracompactness of $M$ we have: 
Proposition 2.2. $\operatorname{Sec}\left(I_{\bigwedge^{p} F^{\prime}}\right)=\left(\operatorname{Sec} I_{F^{\prime}}\right)^{p}, \quad p \geq 1$.

Consider also the symmetric algebra $\bigoplus^{k \geq 0} \operatorname{Sec} \bigvee^{k} F$ and its ideals $\bigoplus^{k \geq p} \operatorname{Sec} \bigvee^{k} F$, $p \geq 1$, and $I_{\text {Sec } F}$. Using the paracompactness of $M$, we can also assert

2.3. $\bigoplus^{k \geq p} \operatorname{Sec} \bigvee^{k} F=\left(I_{\operatorname{Sec} F}\right)^{p}, p \geq 1$.

\section{The CATEgory of Regular Lie algebroids; A SURVEy of EXAmples}

The notion of a Lie algebroid comes from J. Pradines $[\mathrm{P}]$ and was invented for the study of differential groupoids. Let $F$ be a $C^{\infty}$ constant dimensional and involutive distribution on a $C^{\infty}$ Hausdorff paracompact connected manifold $M$. By a regular Lie algebroid over $(M, F)([\mathrm{K} 3],[\mathrm{MA}],[\mathrm{P}])$ we mean a system

$$
(A, \llbracket \cdot, \cdot \rrbracket, \gamma)
$$

consisting of a vector bundle $A$ over $M$ and mappings

$$
\llbracket \cdot, \rrbracket: \operatorname{Sec} A \times \operatorname{Sec} A \rightarrow \operatorname{Sec} A, \quad \gamma: A \rightarrow T M,
$$

such that (i) (Sec $A, \llbracket \cdot, \cdot \rrbracket)$ is a real Lie algebra, (ii) $\gamma$, called by K. Mackenzie $[\mathrm{MA}]$ an anchor, is a homomorphism of vector bundles, and $\operatorname{Im} \gamma=F$, (iii) $\operatorname{Sec} \gamma$ : $\operatorname{Sec} A \rightarrow \mathfrak{X}(M), \xi \mapsto \gamma \circ \xi$, is a homomorphism of Lie algebras, (iv) $\llbracket \xi, f \cdot \eta \rrbracket=$ $f \cdot \llbracket \xi, \eta \rrbracket+(\gamma \circ \xi)(f) \cdot \eta, \xi, \eta \in \operatorname{Sec} A, f \in \Omega^{\circ}(M)$.

In the case when $F=T M$, i.e. when $\gamma: A \rightarrow T M$ is a surjective homomorphism, (3.1) is called a transitive Lie algebroid. Let $A$ and $A^{\prime}$ be two regular Lie algebroids on a manifold $M$. A homomorphism $H: A \longrightarrow A^{\prime}$ of vector bundles is said to be a homomorphism of Lie algebroids if $\gamma^{\prime} \circ H=\gamma$ and $\operatorname{Sec} H: \operatorname{Sec} A \rightarrow \operatorname{Sec} A^{\prime}$ is a homomorphism of Lie algebras.

The short exact sequence

$$
0 \longrightarrow \boldsymbol{g} \hookrightarrow A \stackrel{\gamma}{\longrightarrow} F \longrightarrow 0
$$

is called the Atiyah sequence of (3.1). Each fibre $\boldsymbol{g}_{\mid x}, x \in M$, possesses some natural structure of a Lie algebra defined by $[v, w]:=\llbracket \xi, \eta \rrbracket(x)$, where $\xi, \eta \in \operatorname{Sec} A$ are arbitrarily taken cross-sections of $A$ such that $\xi(x)=v, \nu(x)=w$. For a transitive Lie algebroid (3.1), $\boldsymbol{g}$ is a LAB [K1], [MA].

(a) The following are simple examples of transitive Lie algebroids:

\subsection{Finitely dimensional real Lie algebra $\mathfrak{g}$.}

\subsection{Tangent bundle $T M$ to a manifold $M$.}

\subsection{Trivial Lie algebroid $T M \times \mathfrak{g} \quad[\mathrm{NVQ}]$ where $\mathfrak{g}$ is as in 3.1 .}

\subsection{Bundle of jets $J^{k} T M[\mathrm{~L} 1]$.}

(b) The following are important examples of transitive Lie algebroids:

3.5. The Lie algebroid $A(P)$ of a $G$-principal bundle $P=P(M, G)$. One of the definitions of $A(P)$ is as follows: $A(P)=T P / G$ is the space of orbits of the right action $G$ on $T P$ given by differentials of the right translations (see [L2], [MA], [K1]). The anchor: $\gamma([v])=\pi_{\star}(v)$. The bracket is constructed on the basis of the following observation: for each cross-section $\eta \in \operatorname{Sec} A(P)$, there exists exactly one $C^{\infty}$ right-invariant vector field $\eta^{\prime} \in \mathfrak{X}^{R}(P)$ such that $\left[\eta^{\prime}(z)\right]=\eta(\pi z), z \in P$, and 
the mapping $\operatorname{Sec} A(P) \rightarrow \mathfrak{X}^{R}(P), \eta \mapsto \eta^{\prime}$, is an isomorphism of $\Omega^{\circ}(M)$-modules. The bracket $\llbracket \xi, \eta \rrbracket$ for $\xi, \eta \in \operatorname{Sec} A(P)$ is defined in such a way that $\llbracket \xi, \eta \rrbracket^{\prime}=\left[\xi^{\prime}, \eta^{\prime}\right]$.

The Lie algebroid $A(P)$ is a simpler structure than a principal bundle $P$. Namely, nonisomorphic principal bundles can possess isomorphic Lie algebroids. For example, there exists a nontrivial principal bundle for which the Lie algebroid is trivial (the nontrivial Spin(3)-structure of the trivial principal bundle $\mathbb{R}(5) \times S O(3)$ has this property [K1]). A transitive Lie algebroid is said to be integrable if it arises from a principal bundle.

3.6. General form of a transitive Lie algebroids. Every transitive Lie algebroid is - up to an isomorphism - of the form described below. This was done by K. Mackenzie [MA] and, independently, by J. Kubarski [K1].

Let a system $\left(\boldsymbol{g}, \nabla, \Omega_{b}\right)$ be given, consisting of a LAB $\boldsymbol{g}$ on a manifold $M$, a covariant derivative $\nabla$ in $\boldsymbol{g}$ and a 2 -form $\Omega_{b} \in \Omega^{2}(M, \boldsymbol{g})$ on $M$ with values in $\boldsymbol{g}$, fulfilling the conditions: (1) $\nabla^{2} \sigma=-\left[\Omega_{b}, \sigma\right], \sigma \in \operatorname{Sec} \boldsymbol{g},(2) \nabla_{X}[\sigma, \eta]=\left[\nabla_{X} \sigma, \eta\right]+$ $\left[\sigma, \nabla_{X} \eta\right], X \in \mathfrak{X}(M), \sigma, \eta \in \operatorname{Sec} \boldsymbol{g},(3) \nabla \Omega_{b}=0$. Then $T M \oplus \boldsymbol{g}$ forms a transitive Lie algebroid with the bracket defined by $\llbracket(X, \sigma),(Y, \eta) \rrbracket=\left([X, Y],-\Omega_{b}(X, Y)+\right.$ $\left.\nabla_{X} \eta-\nabla_{X} \sigma+[\sigma, \eta]\right)$, and the anchor being the projection onto the first component.

As Mackenzie noticed [MA], this theorem leads to the first step (called algebraic) of a solution of a long-standing problem of A. Weil: Given a 2-form $\Omega_{b} \in \Omega^{2}(M ; \boldsymbol{g})$, when is it the curvature tensor of a connection in a principal bundle $P(M, G)$ over $M$ with $\boldsymbol{g}$ as the $A d$-associated Lie algebra bundle ? The second (last) step is the theorem $[\mathrm{MA}],[\mathrm{A}-\mathrm{M}]$ giving the integrability obstruction of the constructed Lie algebroid $T M \oplus \boldsymbol{g}$

3.7. The Lie algebroid $C D O(\mathfrak{f})$ of covariant differential operators on a vector bundle $\mathfrak{f}[\mathrm{MA}]$. An isomorphic construction of this object is the Lie algebroid $A(\mathfrak{f})$ of a vector bundle [K3]; here the fibre $A(\mathfrak{f})_{\mid x}$ is the space of linear homomorphisms $l: \operatorname{Sec} \mathfrak{f} \rightarrow \mathfrak{f}_{\mid x}$ such that there exists a vector $u \in T_{x} M$ for which $l(f \cdot \nu)=f(x) \cdot l(\nu)+u(f) \cdot \nu_{x}, f \in \Omega^{\circ}(M), \nu \in$ Sec f. A cross-section $\xi \in \operatorname{Sec} A(\mathfrak{f})$ defines in an evident manner a covariant differential operator in $\mathfrak{f}$. The bracket of cross-sections of $A(\mathfrak{f})$ is defined classically for differential operators. $A(\mathfrak{f})$ is naturally isomorphic to the Lie algebroid of the $G L(V)$-principal bundle ( $V$ is the typical fibre of $\mathfrak{f}$ ) of all repers of $\mathfrak{f}$. Locally, i.e. over some neighbourhood $U$ of a point of $M$, the Lie algebroid $A(\mathfrak{f})_{\mid U}$ is isomorphic to the trivial one $T U \times \operatorname{End}(V)$ $[\mathrm{K} 3,5.4 .4]$.

3.8. The Lie algebroid $A(M, \mathcal{F})$ of a transversally complete foliation $(M, \mathcal{F})$ of a connected Hausdorff paracompact manifold $M$. We recall that a foliation $(M, \mathcal{F})$ is said to be transversally complete ( $T C$ for short) if, at each point $x \in M$, the family $L_{c}(M, \mathcal{F})$ of complete global $\mathcal{F}$-foliate vector fields generates the entire tangent space $T_{x} M$. We add that (a) transversally complete foliations perform a crucial role in the theory of Riemannian foliations [MO2], (b) among them there are ones whose Lie algebroids are not integrable. This last fact - discovered by R. Almeida and P. Molino in 1985 [A-M] — was one of the most important moments in the theory of Lie algebroids.

The first structure theorem of Molino [MO2, Th. 4.2] says that the closures of leaves of a TC-foliation $\mathcal{F}$ form a simple foliation $\mathcal{F}_{b}$, called basic, and are the fibres of a locally trivial basic fibration $\pi_{b}: M \rightarrow W$ onto the Hausdorff paracompact basic manifold $W$. 
Let $Q=T M / E(E=T \mathcal{F})$ be the transverse bundle of $\mathcal{F}$. A cross-section $\xi$ of $Q$ having a foliate vector field as its representative is called a transverse field. Its value at a point $x \in M$ determines uniquely the value at any point $y$ of $\left(L_{b}\right)_{x}$, the leaf of the basic foliation $\mathcal{F}_{b}$ through $x$. Transverse fields play a role analogous to that of right-invariant vector fields on principal bundles. The quotient $A(M, \mathcal{F})=Q / \equiv$ of $Q$ by the relation identifying the values of transverse fields at points of a leaf of $\mathcal{F}_{b}$ [corresponding to the right translations of vectors tangent to a principal bundle] has a natural structure of a vector bundle over $W$. The global cross-sections of $A(M, \mathcal{F})$ correspond to transverse fields. These last yield a Lie algebra; so does $\operatorname{Sec} A(M, \mathcal{F})$. Together with the homomorphism $\gamma: A(M, \mathcal{F}) \rightarrow W$ defined simply by the use of the fibration $\pi_{b}: M \rightarrow W$, we obtain a transitive Lie algebroid.

3.9. The Lie algebroid $A(G ; H)$ of a nonclosed Lie subgroup $H$ of $G$. It is the Lie algebroid of the TC-foliation $\mathcal{F}_{G, H}=\{a H ; a \in G\}$ of left cosets of a Lie group $G$ by a connected Lie subgroup $H \subset G$ [If $H$ is closed, then $A(G ; H)$ is trivial, so this Lie algebroid has a meaning only if $H$ is not closed]. $A(G ; H)$ can be constructed without using the general theory of TC-foliations [K2], since its total space is equal to the space of orbits of the right free action $\bar{R}: Q \times \bar{H} \rightarrow Q$ of the closure $\bar{H}$ of $H$ on the transverse bundle $Q$, such that, for $t \in \bar{H}, \bar{R}_{t}: Q \rightarrow Q$ is an automorphism determined by the differential of the right translation $R_{t}: T G \rightarrow T G$ with the use of the stability of $E$ under $R_{t}$. A cross-section $\xi$ of $Q$ is a transverse field if and only if $\xi$ is invariant with respect to the action $\bar{R}$. Therefore, the relation "三" on $Q$ which gives $A(G ; H)$ is defined by: $\bar{v} \equiv \bar{w} \Leftrightarrow \exists t \in \bar{H}, \bar{R}_{t}(\bar{v})=\bar{w}$.

(c) Examples of nontransitive (in general) Lie algebroids.

3.10. A LAB is a totally nontransitive Lie algebroid [MA].

3.11. Any $C^{\infty}$ constant dimensional involutive distribution $E \subset T M$.

3.12. Any regular Lie algebroid (3.1) over $(M, F)$ and any involutive subdistribution $F^{\prime} \subset F$ determine a new regular Lie algebroid $\left(A^{F^{\prime}}, \llbracket \cdot, \cdot \rrbracket, \gamma^{F^{\prime}}\right)$, this time over $\left(M, F^{\prime}\right)$, in which $A^{F^{\prime}}=\gamma^{-1}\left[F^{\prime}\right], \gamma^{F^{\prime}}=\gamma \mid A^{F^{\prime}}$, whereas the bracket of cross-sections of $A^{F^{\prime}}$ forms a Lie subalgebra of $\operatorname{Sec} A$.

\subsection{Any Lie equation $R \subset J^{k}(T M)$.}

3.14. The Lie algebroid $\left(T^{\star} M, \llbracket \cdot, \cdot \rrbracket, \gamma\right)$ of a Poisson manifold $(M,\{\cdot, \cdot\})$ $[\mathrm{C}-\mathrm{D}-\mathrm{W}]$. The bracket $\llbracket \cdot, \cdot \rrbracket$ of 1 -forms is defined uniquely by demanding that $\llbracket d f, d g \rrbracket=d\{f, g\}$ for $f, g \in \Omega^{\circ}(M)$, whereas the anchor $\gamma(d f)$ is equal to the Hamiltonian vector field corresponding to $f$.

3.15. The Lie algebroid $i^{\star} T^{\alpha} \Phi$ of a differential groupoid $\Phi[\mathrm{P}]$. Here $T^{\alpha} \Phi$ is the vector bundle of $\alpha$-vertical vectors, while $i: M \hookrightarrow \Phi$ is the inclusion of the manifold of units. Particularly, we have:

3.16. The Lie algebroid of an action $T: M \times G \rightarrow M$ of a Lie group $G$ on $M$, because $T$ determines a structure of a differential groupoid on $M \times G$ with the source $\alpha(x, g)=x$, the target $\beta(x, g)=x \cdot g$, and the partial multiplication $(x, g) \cdot(x \cdot g, h)=(x, g \cdot h)$.

\section{Partial connections in Regular Lie algebroids}

By a phconnection in a regular Lie algebroid $(3.1)$ over $(M, F)$ we mean a homomorphism of vector bundles $\lambda: F \rightarrow A$, such that $\gamma \circ \lambda=i d_{F}$, i.e. any splitting 
of the Atiyah sequence (3.2) of (3.1). The uniquely determined homomorphism $\omega: A \rightarrow \boldsymbol{g}$ for which $\omega \mid \boldsymbol{g}=i d$ and $\omega \mid \operatorname{Im} \lambda=0$ is called the connection form of $\lambda$. By the curvature tensor of $\lambda$ we shall mean the tangential differential 2 -form $\Omega_{b} \in \Omega_{F}^{2}(M ; \boldsymbol{g})$ defined by

$$
\Omega_{b}\left(X_{1}, X_{2}\right)=-\omega\left(\llbracket \lambda \circ X_{1}, \lambda \circ X_{2} \rrbracket\right), \quad X_{i} \in \operatorname{Sec} F .
$$

We recall that the space $\Omega_{F}^{k}(M ; \boldsymbol{g})$ of tangential differential $k$-forms on $(M, F)$ with values in $\boldsymbol{g}$ is the space of cross-sections of the vector bundle $\bigwedge^{k} F^{\star} \otimes \boldsymbol{g}$.

Definition 4.1. Let $(A, \llbracket \cdot, \cdot \rrbracket, \gamma)$ be a regular Lie algebroid over $(M, F)$. By a partial connection in $A$ over $F^{\prime}, F^{\prime}$ being an involutive subbundle of $F$, we mean any linear homomorphism $\lambda^{\prime}: F^{\prime} \rightarrow A$ such that $\gamma \circ \lambda^{\prime}=i d_{F^{\prime}}$, i.e. equivalently, any connection in the regular Lie algebroid $A^{F^{\prime}}:=\gamma^{-1}\left[F^{\prime}\right]$ over $\left(M, F^{\prime}\right)$ (see Ex. $3.12)$.

Remark 4.2. The term "partial connection" comes from the theory of foliated principal bundles $[\mathrm{K}-\mathrm{T}]$ and means, for a given principal bundle $P$, some right-invariant horizontal subbundle of $T P$ projectable onto a distribution $F^{\prime}$ on $M$. Besides, there is a one-to-one correspondence between partial connections in $P$ over an involutive distribution $F^{\prime}$ and partial connections over $F^{\prime}$ in the Lie algebroid $A(P)$ of $P$. Via this correspondence, to a flat connection there corresponds a flat one. This correspondence looks as follows: with a connection $\lambda: F \rightarrow A(P)^{F}$ we associate a partial connection $H^{\lambda} \subset T P$ projectable onto $F$, defined by $H_{\mid z}^{\lambda}=\left(\pi_{\mid z}^{A}\right)^{-1}\left[\operatorname{Im} \lambda_{\pi z}\right]$, $z \in P$, where $\pi: P \rightarrow M$ and $\pi^{A}: T P \rightarrow A(P)$ are the canonical projections. The observation concerning the equivalence of the flatness of $\lambda$ and $H^{\lambda}$ follows from the relation

$$
\Omega_{b}(x ; v \wedge w)=\hat{z}\left(\Omega\left(z ; v^{z} \wedge w^{z}\right)\right), \quad v, w \in F_{\mid \pi z},
$$

between the curvature tensor $\Omega_{b} \in \Omega_{F}^{2}(M ; \boldsymbol{g})$ of $\lambda$ and the curvature form $\Omega \in$ $\Omega^{2}(P ; \mathfrak{g})$ of any connection in $P$ adapted to $H^{\lambda}$ ( $\mathfrak{g}$ is the Lie algebra of the structure Lie group of $P$ ). In this relation $v^{z}$ denotes the horizontal lifting of $v$, whereas $\hat{z}: \mathfrak{g} \rightarrow \boldsymbol{g}_{\mid \pi z}$ is an isomorphism defined by $\hat{z}(u)=\left[\left(A_{z}\right)_{\star e}(u)\right], u \in \mathfrak{g}$, where $A_{z}: G \rightarrow P, a \mapsto z \cdot a$. We add that $\hat{z}$ is an isomorphism of Lie algebras provided that in $\mathfrak{g}$ we have the right Lie algebra structure of $G$.

A connection $\lambda: F \rightarrow A$ in a regular Lie algebroid (3.1) over $(M, F)$ is said to be adapted to a given partial connection $\lambda^{\prime}$ over $F^{\prime} \subset F$ if $\lambda^{\prime}=\lambda \mid F^{\prime}$. It is evident that an adapted connection always exists.

Consider in a regular Lie algebroid $A$ over $(M, F)$ a connection $\lambda$ and a partial connection $\lambda^{\prime}$ over $F^{\prime}$. Let $\omega$ and $\omega^{\prime}$ be the connection forms of $\lambda$ and $\lambda^{\prime}$, respectively, and let $\Omega_{b}$ and $\Omega_{b}^{\prime}$ be the curvature tensors of these connections.

Proposition 4.3. If $\lambda$ is adapted to $\lambda^{\prime}$ and $j_{o}: F^{\prime} \hookrightarrow F$ is an inclusion, then $\Omega_{b}^{\prime}=j_{o}^{\star} \Omega_{b}$, where $j_{o}^{\star} \Omega_{b}$ is defined by the obvious formula

$$
j_{o}^{\star} \Omega_{b}(X, Y)=\Omega_{b}\left(j_{o} \circ X, j_{o} \circ Y\right) .
$$

Proposition 4.4. If $\lambda$ is adapted to $\lambda^{\prime}$, then the following conditions are equivalent:

1. $\lambda^{\prime}$ is flat,

2. $j_{o}^{\star} \Omega_{b}=0$,

3. $i(a)\left(\left\langle v^{\star}, \Omega_{b \mid x}\right\rangle\right)=0$ for all $x \in M, a \in \bigwedge^{2} F_{\mid x}^{\prime}$, and $v^{\star} \in \boldsymbol{g}_{\mid x}^{\star}$, 
4. $\left\langle v^{\star}, \Omega_{b \mid x}\right\rangle \in I_{F_{\mid x}^{\prime \perp}}^{(2)}$ for all $x \in M$ and $v^{\star} \in \boldsymbol{g}_{\mid x}^{\star}$.

Proof. $(1) \Leftrightarrow(2)$ is evident by $4.3 . \quad(2) \Leftrightarrow(3)$ is trivial, whereas $(3) \Leftrightarrow(4)$ follows from 2.1.

Any foliated principal bundle $[\mathrm{K}-\mathrm{T}]$ determines, clearly, a transitive Lie algebroid with a flat partial connection.

Definition 4.5. A connection $\lambda$ adapted to $\lambda^{\prime}$ is said to be basic if

$$
i_{X} \Omega_{b}=0 \text { for all } X \in \operatorname{Sec} F^{\prime} .
$$

Proposition 4.6. Let a connection $\lambda$ be adapted to $\lambda^{\prime}$. Then

A) the following conditions are equivalent:

(1) $\lambda$ is basic,

(2) $i(a)\left(\left\langle v^{\star}, \Omega_{b \mid x}\right\rangle\right)=0$ for all $x \in M, a \in F_{\mid x}^{\prime}$, and $v^{\star} \in \boldsymbol{g}_{\mid x}^{\star}$,

(3) $\left\langle v^{\star}, \Omega_{b \mid x}\right\rangle \in I_{\bigwedge^{2} F_{\mid x}^{\prime \perp}}^{(2)}$ for all $x \in M$ and $v^{\star} \in \boldsymbol{g}_{\mid x}^{\star}$;

B) if $\lambda$ is basic, then $\lambda^{\prime}$ is flat.

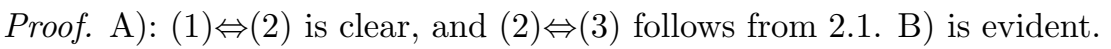

Remark 4.7. We describe here shortly some obstructions to the existence of a basic connection. Assume that $\lambda$ is adapted to $\lambda^{\prime}$. This produces an exact short sequence of vector bundles

$$
0 \longrightarrow \boldsymbol{g} \hookrightarrow A / \operatorname{Im} \lambda^{\prime} \longrightarrow F / F^{\prime} \longrightarrow 0
$$

whose splittings are in 1-1 correspondence to adapted connections. More precisely, $\bar{\omega} \mapsto \bar{\omega} \circ c$ (where $c: A \rightarrow A / \operatorname{Im} \lambda^{\prime}$ is the canonical projection) establishes this correspondence. Passing to spaces of cross-sections and using the paracompactness of $M$, we get the following exact short sequence of $\Omega^{\circ}(M)$-modules:

$$
0 \longrightarrow \operatorname{Sec} \boldsymbol{g} \hookrightarrow \operatorname{Sec}\left(A / \operatorname{Im} \lambda^{\prime}\right) \longrightarrow \operatorname{Sec}\left(F / F^{\prime}\right) \longrightarrow 0 .
$$

There are representations of the Lie algebra Sec $F^{\prime}$ in all $\Omega^{\circ}(M)$-modules occurring in (4.2), namely, for $X^{\prime} \in \operatorname{Sec} F^{\prime}$, they are defined by

(a) $\left(X^{\prime}, \nu\right) \mapsto \llbracket \lambda \circ X^{\prime}, \nu \rrbracket, \nu \in \operatorname{Sec} \boldsymbol{g}$

(b) $\left(X^{\prime},[\xi]\right) \mapsto\left[\llbracket \lambda \circ X^{\prime}, \xi \rrbracket\right], \quad[\xi] \in \operatorname{Sec}\left(A / \operatorname{Im} \lambda^{\prime}\right)$ for $\xi \in \operatorname{Sec} A$,

(c) $\left(X^{\prime},[Y]\right) \mapsto\left[\left[X^{\prime}, Y\right]\right], \quad[Y] \in \operatorname{Sec}\left(F / F^{\prime}\right)$ for $Y \in \operatorname{Sec} F$.

One can prove that

- a basic connection exists if and only if

$\left.{ }^{*}\right)$ sequence (4.2) has a splitting in the category of $\Omega^{\circ}(M)$-modules, being equivariant with respect to the above representations.

More precisely, for a splitting $\bar{\omega}$ of $(4.1), \bar{\omega} \circ c$ is a basic connection form if and only if $\operatorname{Sec} \bar{\omega}: \operatorname{Sec}\left(A / \operatorname{Im} \lambda^{\prime}\right) \rightarrow \operatorname{Sec} \boldsymbol{g}$ is an equivariant homomorphism.

Condition $\left(^{*}\right)$ can be equivalently formulated in the terms of the vanishing of a suitable element of some sheaf cohomology group.

\section{Bott's Vanishing Theorem for Regular Lie algebroids}

The aim of this section is to prove the following theorem concerning the Pontryagin algebra $\operatorname{Pont}(A)$ of a regular Lie algebroid $A$. 
Theorem 5.1 (Bott's Vanishing Theorem). Let (3.1) be a regular Lie algebroid over $(M, F)$. If there exists a flat partial connection $\lambda^{\prime}$ in $A$ over $F^{\prime} \subset F$, then

$$
\operatorname{Pont}^{p}(A)=0 \quad \text { for } \quad p \geq 2 \cdot(q+1)
$$

where $q=\operatorname{rank}\left(F / F^{\prime}\right)$. If, moreover, $\lambda^{\prime}$ admits a basic adapted connection, then

$$
\operatorname{Pont}^{p}(A)=0 \quad \text { for } \quad p \geq q+1 .
$$

Proof. (i) If $\lambda^{\prime}$ is a partial connection in $A$ over $F^{\prime}$, then, for each point $x \in M$, the algebra $\bigwedge F_{\mid x}^{\star}$ possesses a decreasing filtration by ideals

$$
\mathrm{F}^{p}\left(\bigwedge F_{\mid x}^{\star}\right)=I \bigwedge^{p}\left(F_{\mid x}^{\prime \perp}\right), \quad p \geq 1
$$

which determines the decreasing filtration by ideals of the algebra $\Omega_{F}(M)$ :

$$
\begin{aligned}
\mathrm{F}^{p}\left(\Omega_{F}(M)\right) & =\left\{\Theta \in \Omega_{F}(M) ; \forall x \in M, \Theta_{x} \in \mathrm{F}^{p}\left(\bigwedge F_{\mid x}^{\star}\right)\right\} \\
( & =\operatorname{Sec}\left(I_{\bigwedge^{p}\left(F^{\prime \perp}\right)}\right) .
\end{aligned}
$$

(ii) For an arbitrary connection $\lambda$ in $A$, consider the homomorphism of algebras [K3, s.4.1]

$$
\begin{aligned}
\chi_{(A, \lambda)}: I^{\circ}(A) & \longrightarrow \Omega_{F}(M), \\
I^{\circ k}(A) \ni \Gamma & \longmapsto \frac{1}{k !} \cdot\langle\Gamma, \underbrace{\Omega_{b} \vee \cdots \vee \Omega_{b}}_{k \text { times }}\rangle,
\end{aligned}
$$

where $\Omega_{b} \in \Omega_{F}^{2}(M)$ is the curvature tensor of $\lambda$, whereas

$$
\Omega_{b} \vee \cdots \vee \Omega_{b} \in \Omega_{F}^{2 k}\left(M ; \bigvee^{k} \boldsymbol{g}^{\star}\right)
$$

is defined by the formula

$$
\begin{aligned}
& \Omega_{b} \vee \cdots \vee \Omega_{b}\left(x ; v_{1} \wedge \cdots \wedge v_{2 k}\right) \\
& =\frac{1}{2^{k}} \cdot \sum_{\sigma} \operatorname{sgn} \sigma \cdot \Omega_{b}\left(x ; v_{\sigma(1)} \wedge v_{\sigma(2)}\right) \vee \cdots \vee \Omega_{b}\left(x ; v_{\sigma(2 k-1)} \wedge v_{\sigma(2 k)}\right) .
\end{aligned}
$$

The algebra $I^{\circ}(A)$ has a standard even decreasing filtration by ideals

$$
\mathrm{F}^{2 p}\left(I^{\circ}(A)\right)=\bigoplus^{k \geq p} \operatorname{Sec} \bigvee^{k} \boldsymbol{g}^{\star}, \quad p \geq 1 .
$$

(iii) The crucial part of the proof of our theorem is based on the following:

Lemma 5.2. If $\lambda$ is a connection in $A$ adapted to a flat partial connection $\lambda^{\prime}$ over $F^{\prime}$, then the homomorphism $\chi_{(A, \lambda)}$ is filtration-preserving in the sense that

$$
\chi_{(A, \lambda)}\left[\mathrm{F}^{2 p}\left(I^{\circ}(A)\right)\right] \subset \mathrm{F}^{p}\left(\Omega_{F}(M)\right), \quad p \geq 1 ;
$$

if $\lambda$ is, in addition, basic, then

$$
\chi_{(A, \lambda)}\left[\mathrm{F}^{2 p}\left(I^{\circ}(A)\right)\right] \subset \mathrm{F}^{2 p}\left(\Omega_{F}(M)\right), \quad p \geq 1 .
$$

Proof of the lemma. Because of the fact that $\chi_{(A, \lambda)}$ is a homomorphism of algebras, and that the ideals $\mathrm{F}^{2 p}\left(I^{\circ}(A)\right), \mathrm{F}^{p}\left(\Omega_{F}(M)\right)$ and $\mathrm{F}^{2 p}\left(\Omega_{F}(M)\right)$ are the $p$-th powers of the ideals $I_{\operatorname{Sec} \boldsymbol{g}^{\star}}$, $\operatorname{Sec}\left(I_{F^{\prime} \perp}\right)$ and $\operatorname{Sec}\left(I_{\Lambda^{2} F^{\prime \perp}}\right)$, respectively, see Propositions $2.2-2.3$, it suffices to check that

(a) $\chi_{(A, \lambda)}\left[\operatorname{Sec} \boldsymbol{g}^{\star}\right] \subset \operatorname{Sec}\left(I_{F^{\prime} \perp}\right)$,

(b) for a basic connection,

$$
\chi_{(A, \lambda)}\left[\operatorname{Sec} \boldsymbol{g}^{\star}\right] \subset \operatorname{Sec}\left(I_{\bigwedge^{2} F^{\prime \perp}}\right) .
$$


However, for $\Gamma \in \operatorname{Sec} \boldsymbol{g}^{\star}, \chi_{(A, \lambda)}(\Gamma)_{\mid x}=\left\langle\Gamma_{x}, \Omega_{b \mid x}\right\rangle$, and so (a) and (b) follow from 4.4 and 4.6, respectively, which ends the proof of our lemma.

(iv) To prove our theorem, assume that in $A$ there is a flat partial connection, say $\lambda^{\prime}$, over $F^{\prime}$. Take arbitrarily an adapted connection $\lambda$. If $q=\operatorname{rank}\left(F / F^{\prime}\right)(=$ $\operatorname{rank} F^{\prime \perp}$ ), we have a simple but important observation:

$$
\bigwedge^{p}\left(F^{\prime \perp}\right)=0 \quad \text { for } \quad p \geq q+1 .
$$

In consequence,

$$
\mathrm{F}^{p}\left(\Omega_{F}(M)\right)=0 \text { for } \quad p \geq q+1 .
$$

Lemma 5.2 now implies in our situation the relation

$$
\chi_{(A, \lambda)}\left[\mathrm{F}^{2 p}\left(I^{\circ}(A)\right)\right]=0
$$

for $p \geq q+1$, and under the additional assumption that $\lambda$ is basic, such a relation for $2 p \geq q+1$, i.e. for $p \geq[q / 2]+1$. Passing to cohomologies, we obtain our theorem because $\operatorname{Pont}^{p}(A)=H_{F}^{p}(M) \cap \operatorname{Im} h_{A}$ and the Chern-Weil homomorphism $h_{A}$ is defined on the level of forms via $\chi_{(A, \lambda)}$ for an arbitrarily taken connection $\lambda$.

Remark 5.3. For $A=A(P), P$ being a connected principal bundle, we obtain the classical Bott's Vanishing Theorem for foliated principal bundles [K-T] because

(a) between connections in $A$ and connections in $P$ there exists a bijection which maps connections in $A$, flat over a given distribution $F \subset T M$, into connections flat over this distribution (see Remark 4.2),

(b) the Chern-Weil homomorphisms $h_{A}$ and $h_{P}$ are equivalent in the sense of Th.1.1 (particularly, $\operatorname{Pont}(P)=\operatorname{Pont}(A)$ ).

Remark 5.4. The following statement is an immediate corollary from Th.5.1:

- If $\operatorname{Pont}^{p}(A) \neq 0$, then there exists no flat partial connection $\lambda^{\prime}$ in $A$ over any involutive distribution $F^{\prime} \subset F$ such that

$$
\operatorname{rank} F^{\prime} \geq \operatorname{rank} F+1-p / 2,
$$

and, moreover, such that $\lambda^{\prime}$ - additionally - admits a basic adapted connection and

$$
\operatorname{rank} F^{\prime} \geq \operatorname{rank} F+1-p
$$

Remark 5.5. When seeking for an involutive subdistribution $F^{\prime \prime} \subset F$ over which there exists a flat partial connection and which is contained in a given involutive subdistribution $F^{\prime} \subset F$, some obstructions in lower dimensional cohomology groups appear. Namely, we have

$$
\operatorname{Pont}^{p}\left(A^{F^{\prime}}\right)=0 \quad \text { for } \quad p \geq 2\left(q+1-\operatorname{rank}\left(F / F^{\prime}\right)\right)
$$

where, of course, $q=\operatorname{rank}\left(F / F^{\prime \prime}\right)$. However, they are in the tangential cohomology groups $H_{F^{\prime}}(M)$ on $M$ with respect to $F^{\prime}$, not to $F$. On the other hand, in [K4] there is a suggestion that the vanishing of $\operatorname{Pont}^{p}(A)$ does not imply, in general, the vanishing of $\operatorname{Pont}^{p}\left(A^{F^{\prime}}\right)$, which may be, additionally, a new impulse to the consideration of the Pontryagin algebra $\operatorname{Pont}\left(A^{F^{\prime}}\right)$ instead of $\operatorname{Pont}(A)$.

As a more concrete example, consider some nonorientable Riemannian vector bundle $\mathfrak{f}$ of rank $2 m$ and the connected $O(2 m ; R)$-principal bundle $P$ of orthonormal repers of $\mathfrak{f}$, corresponding to it, and further, the transitive Lie algebroid $A=A(P)$. We have Pont ${ }^{2 m}(P)=0$ (and, of course, Pont $^{k}(P)=0$ for $k>2 m$ ). In [K4] there 
is a conjecture that there exists an example of $\mathfrak{f}$ and an involutive distribution $F^{\prime}$ on the base $M$ of $\mathfrak{f}$ for which

$$
\operatorname{Pont}^{2 m}\left(A^{F^{\prime}}\right) \neq 0 \text {. }
$$

Applying Th.5.1 to the Lie algebroid $A^{F^{\prime}}$, we obtain that, under condition (5.1), - there is no involutive subdistribution $F^{\prime \prime} \subset F^{\prime}$ over which there exists a flat partial Riemannian connection in $\mathfrak{f}$, such that $2 m \geq 2\left(\operatorname{rank}\left(F^{\prime} / F^{\prime \prime}\right)+1\right)$, i.e. equivalently, that

$$
\operatorname{rank} F^{\prime \prime} \geq \operatorname{rank} F^{\prime}+1-m \text {. }
$$

\section{BotT'S PHENOMENON FOR TC-FOLIATIONS}

Every transversally complete foliation $(M, \mathcal{F})$ possesses a transitive Lie algebroid $A(M, \mathcal{F})$ (see [MO1], [MO2] and, for more details, [K3]).

- What does Bott's Vanishing Theorem assert for a TC-foliation?

To answer this question, we need to explain the notion of a flat partial connection in $A(M, \mathcal{F})$ in the language of the foliation $(M, \mathcal{F})$.

Let $(M, \mathcal{F})$ be a given TC-foliation of a connected Hausdorff and paracompact manifold $M$, see Ex. 3.8. Let $E$ and $E_{b}$ be the vector bundles tangent to $\mathcal{F}$ and to the basic foliation $\mathcal{F}_{b}$, respectively; let $L(M, \mathcal{F})$ and $l(M, \mathcal{F})$ denote the Lie algebras of foliate vector fields and transverse fields, respectively; whereas $\alpha: T M \rightarrow Q$ and $\beta: Q \rightarrow A(M, \mathcal{F})$ denote the canonical projections. In [K3] there is obtained a canonical bijection

$$
\lambda \longmapsto \bar{C}^{\lambda}:=\alpha^{-1}\left[\beta^{-1}[\operatorname{Im} \lambda]\right]
$$

between connections in $A(M, \mathcal{F})$ and distributions $\bar{C} \subset T M$ such that (i) $E_{b} \cap \bar{C}=$ $E$, (ii) $E_{b}+\bar{C}=T M$, (iii) $L(M, \mathcal{F}) \cap \operatorname{Sec} \bar{C}$ generates at each point $x \in M$ the entire vector space $\bar{C}_{\mid x}$. We add that a connection $\lambda$ is flat if and only if the corresponding distribution $\bar{C}^{\lambda}$ is involutive.

A distribution $\bar{C}$ fulfilling (i)-(iii) above is called an $\mathcal{F}$ - connection (or a connection in $T M$ for the $T C$-foliation $(M, \mathcal{F})$ ).

For any distribution $F$ on the basic manifold $W$ we define a distribution $\bar{F}$ on $M$ by

$$
\bar{F}=\alpha^{-1}\left[\beta^{-1}\left[\gamma^{-1}[F]\right]\right] \quad\left(=\pi_{b \star}{ }^{-1}[F]\right),
$$

$\gamma$ being the anchor in $A(M, \mathcal{F})$. Notice that $\operatorname{rank} \bar{F}=\operatorname{rank} F+r_{b} \quad r_{b}=\operatorname{dim} \mathcal{F}_{b}$. Clearly, we have

6.1. The correspondence $F \rightarrow \bar{F}$ establishes a bijection between involutive $C^{\infty}$ distributions on $W$ and distributions $\bar{F}$ on $M$ such that (a) $\bar{F}$ is involutive, (b) $E_{b} \subset \bar{F}$, (c) $L(M, \mathcal{F}) \cap \operatorname{Sec} \bar{F}$ generates at each point $x \in M$ the entire vector space $\bar{F}_{\mid x}$.

Each distribution on $M$ satisfying (a)-(c) above is called an $\mathcal{F}$-distribution.

Now, pass to partial connections. Let $F \subset T W$ be any involutive distribution on $W$ and $\lambda: F \rightarrow A(M, \mathcal{F})^{F}$ any partial connection in $A(M, \mathcal{F})$ over $F$. Put $\bar{C}^{\lambda}:=\alpha^{-1}\left[\beta^{-1}[\operatorname{Im} \lambda]\right]$.

Proposition 6.2. The correspondence $\lambda \mapsto \bar{C}^{\lambda}$ establishes a bijection between partial connections in $A(M, \mathcal{F})$ over $F$ and distributions $\bar{C} \subset T M$ such that (i) $E_{b} \cap \bar{C}=E$, (ii) $E_{b}+\bar{C}=\bar{F}$, (iii) $L(M, \mathcal{F}) \cap \operatorname{Sec} \bar{C}$ generates at each point $x \in M$ the entire vector space $\bar{C}_{\mid x}$. 
In particular, such a distribution $\bar{C}$ exists and is $C^{\infty}$. A partial connection $\lambda$ is flat if and only if the corresponding distribution $\bar{C}^{\lambda}$ is involutive.

Proof. The bijectivity of the correspondence is easy to obtain. To prove that a flat partial connection passes to an involutive distribution (and vice versa), take the connection form $\omega: A(M, \mathcal{F})^{F} \rightarrow \boldsymbol{g}$ and the curvature tensor $\Omega_{b} \in \Omega_{F}^{2}(W ; \boldsymbol{g})$ of a partial connection $\lambda$ over $F$. Next, analogously to [K3, Def.6.3.2], we define the so-called connection form $\bar{\omega}: \bar{F} \rightarrow Q^{\prime}$ and curvature form $\bar{\Omega} \in \Omega_{\bar{F}}^{2}\left(M ; Q^{\prime}\right)$ of $\bar{C}^{\lambda}\left(Q^{\prime}:=E_{b} / E\right)$ in such a way that diagrams

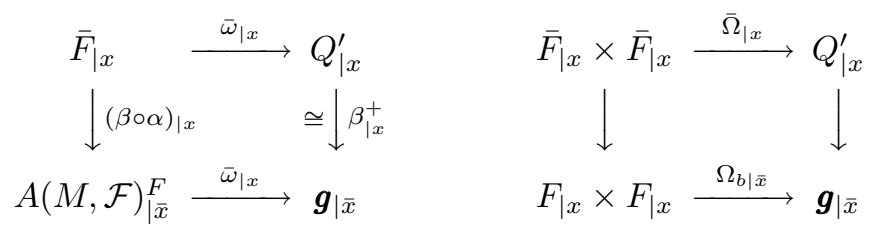

commute for each $x \in M\left(\bar{x}:=\pi_{b}(x)\right)$. Analogously to $[K 3$, s.6.3.4] we prove that

$$
\Omega\left(Y_{1}, Y_{2}\right)=-\bar{\omega}\left(\left[K \circ Y_{1}, K \circ Y_{2}\right]\right)
$$

for $Y_{i} \in L(M, \mathcal{F})$, where $K: \bar{F} \rightarrow \bar{F}$ is the horizontal projection defined as the projection onto the second component with respect to the decomposition $\bar{F}=$ $E_{b} \oplus \bar{C}_{u}$, where $\bar{C}_{u} \subset \bar{C}^{\lambda}$ is an arbitrarily taken subbundle such that $\bar{C}^{\lambda}=E \oplus \bar{C}_{u}$. Next, using (6.2), we can prove the equivalence of the conditions: (i) $\Omega_{b}=0$, (ii) $\bar{\Omega}=0$, (iii) $L(M, \mathcal{F}) \cap \operatorname{Sec} \bar{C}$ is a Lie subalgebra of $L(M, \mathcal{F})$, (iv) $\bar{C}^{\lambda}$ is involutive. This ends the proof.

Each distribution $\bar{C}$ satisfying (i)-(iii) from Proposition 6.2 is called a partial $\mathcal{F}$-connection (or a partial connection for $(M, \mathcal{F})$ ) over $\bar{F}$.

Since the codimensions of the foliations determined by $F$ and $\bar{F}$ are equal to each other, we obtain (as a corollary from 5.1) the following

Corollary 6.3 (Bott's Vanishing Theorem for TC-foliations). If there exists an involutive distribution $\bar{C}$ on $M$ satisfying (i)-(iii) from 6.2 for a distribution $\bar{F}$ on $M$ satisfying (a)-(c) from 6.1 , then

$$
\operatorname{Pont}^{p}(A(M, \mathcal{F}))=0, \quad p \geq 2 \cdot(q+1),
$$

where $q=\operatorname{codim} \bar{F}$.

\section{BotT'S PHENOMENON FOR NONCLOSED LIE SUBGROUPS}

Let $H$ be a connected Lie subgroup of a connected Lie group $G$ and $A(G ; H)$ its Lie algebroid (see Ex.3.9). We start with a technical lemma.

Lemma 7.1. Let $\bar{C} \subset T G$ be a $C^{\infty}$ distribution on $G$ containing $E$. Then the condition

$\left.{ }^{*}\right) L(G, \mathcal{F}) \cap \operatorname{Sec} \bar{C}$ generates at each point $g \in G$ the entire vector space $\bar{C}_{\mid g}$ is equivalent to

(**) $\bar{C}$ is stable under the action $R: T G \times \bar{H} \rightarrow T G$ of $\bar{H}$ on $T G$ by differentials of the right translations on $G$.

Proof. $\left({ }^{*}\right) \Rightarrow\left({ }^{* *}\right)$. Take arbitrarily $g \in G$ and $v \in \bar{C}_{\mid g}$. Find a vector field $X \in$ $L\left(G, \mathcal{F}_{G ; H}\right) \cap \operatorname{Sec} \bar{C}$ such that $X_{g}=v$. Since the cross-section $\bar{X}$ of $Q$ corresponding to $X$ is a transverse field, we have, for $t \in \bar{H}, \quad \bar{X}_{g t}=\bar{R}_{t}\left(\bar{X}_{g}\right)$, i.e. $X_{g t}-R_{t}\left(X_{g}\right) \in$ $E_{\mid g t}$. The relation $X_{g t} \in \bar{C}_{\mid g t}$ implies $R_{t}(v)=R_{t}\left(X_{g}\right) \in \bar{C}_{\mid g t}$. 
$(* *) \Rightarrow\left({ }^{*}\right)$. Assume $\bar{C}$ to be $R$-stable. $\bar{C}$ determines a $C^{\infty}$ vector subbundle $C^{\prime}=\bar{C} / E \subset Q$ which is $\bar{R}$-stable. This implies that there exists a vector subbundle $C \subset A(G ; H)$ such that $C_{\mid \bar{g}}=\beta_{\mid g}\left[\bar{C}_{\mid g}\right], \bar{g}:=\pi_{b}(g) \quad(\beta: Q \rightarrow A(G ; H)$ is the canonical projection). Reasoning similar to that in Step 3 of the proof of Th.3.4 from [K2] gives that $C$ is $C^{\infty}$. To prove that condition (*) holds, take $v \in \bar{C}_{\mid g}$ and put $u:=[\bar{v}] \in A(G ; H)_{\mid \bar{g}}([\bar{v}]:=\beta \circ \alpha(v))$. Since $u \in C_{\mid \bar{g}}$ and $C$ is a $C^{\infty}$ vector bundle, there is a $C^{\infty}$ cross-section $\xi$ of $C$ such that $\xi_{\bar{g}}=u$. Next, there exists a foliate vector field $X \in L(G, \mathcal{F})$ which determines $\xi$. By the construction, $X \in L(G, \mathcal{F}) \cap \operatorname{Sec} \bar{C}$ and $X_{g}-v \in E_{\mid g}$. Adding, if necessary, a suitable vector field from $\mathfrak{X}(\mathcal{F})$ to $X$, we obtain a vector field which is foliate, lying in $\bar{C}$, and has at $g$ the vector $v$ as its value.

As consequences we obtain a number of corollaries.

Corollary 7.2. $A C^{\infty}$ distribution $\bar{C}$ on $G$ is an $\mathcal{F}$-connection if and only if (i) $E_{b} \cap \bar{C}=E$, (ii) $E_{b}+\bar{C}=T G$, (iii) $\bar{C}$ is $R$-stable.

Corollary 7.3. A $C^{\infty}$ distribution $\bar{F}$ on $G$ is an $\mathcal{F}$-distribution if and only if (a) $\bar{F}$ is involutive, (b) $E_{b} \subset \bar{F}$, (c) $\bar{F}$ is R-stable.

Corollary 7.4. A $C^{\infty}$ distribution $\bar{C}$ on $G$ is a partial $\mathcal{F}$-connection over an $\mathcal{F}$ distribution $\bar{F}$ if and only if (i) $E_{b} \cap \bar{C}=E$, (ii) $E_{b}+\bar{C}=\bar{F}$, (iii) $\bar{C}$ is R-stable.

In [K3, Ex.7.4.7] it is shown that the $G$-left-invariant distribution $\bar{C}$ on $G$ generated by a Lie subalgebra $\mathfrak{c} \subset \mathfrak{g}$ of $\mathfrak{g}$ such that (i) $\mathfrak{c}+\overline{\mathfrak{h}}=\mathfrak{g}$, (ii) $\mathfrak{c} \cap \overline{\mathfrak{h}}=\mathfrak{h}$, is

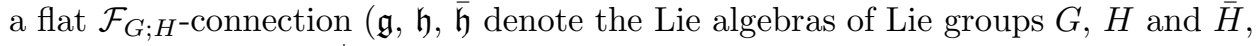
respectively). Since $h_{A(G ; H)}^{+} \neq 0$ for $G$ a compact and semisimple Lie group and $H$ nonclosed [K3, Th.7.4.3], we obtain that no Lie subalgebra $\mathfrak{c}$, satisfying (i) and (ii) above, exists.

In the cited work [K3], in fact, the more detailed result, namely, $h_{A(G ; H)}^{2} \neq 0$ is proved. See also [K2] where it is shown that the Lie group $U(2)$ possesses a nonclosed connected Lie subgroup $H$ for which $h_{A(U(2) ; H)}^{2} \neq 0$. Such results are the sources of some strengthening of Cor.7.4.7 from [K3], basing on Bott's phenomenon.

We start giving some examples of $\mathcal{F}$-distributions on $G$. We see without difficulty the following lemma.

Lemma 7.5. (1). If $\mathfrak{f} \subset \mathfrak{g}$ is a Lie subalgebra of $\mathfrak{g}$ such that $\overline{\mathfrak{h}} \subset \mathfrak{f}$ then the $G$-leftinvariant distribution $\bar{F}(\mathfrak{f}) \subset T G$ determined by $\mathfrak{f}$ is an $\mathcal{F}$-distribution. (2). The corresponding involutive distribution $F(\mathfrak{f}) \subset T(G / \bar{H})$ on the homogeneous space $G / \bar{H}$ is $G$-left-invariant (with respect to the standard left action of $G$ on $G / \bar{H}$ by left translations) and generated by $\mathfrak{f} / \overline{\mathfrak{h}}$. Besides, $\operatorname{codim} F(\mathfrak{f})=\operatorname{codim} \mathfrak{f}$.

Some Lie subalgebras of $G$ are the sources of partial connections in $A(G ; H)$.

Lemma 7.6. If $\tilde{\mathfrak{c}} \subset \mathfrak{g}$ is a vector subspace of $\mathfrak{g}$ such that

(i) $\tilde{\mathfrak{c}} \cap \overline{\mathfrak{h}}=\mathfrak{h}$,

(ii) $\tilde{\mathfrak{c}}+\overline{\mathfrak{h}}$ is a Lie subalgebra of $\mathfrak{g}$, say $\mathfrak{f}$,

(iii) $A d_{G}(t)[\tilde{\mathfrak{c}}] \subset \tilde{\mathfrak{c}}$ for $t \in H$,

then the $G$-left-invariant distribution $\bar{C}(\tilde{\mathfrak{c}})$ generated by $\tilde{\mathfrak{c}}$ is a partial $\mathcal{F}$-connection over $\bar{F}(\mathfrak{f})$. The partial connection in $A(G ; H)$ over $F(\mathfrak{f})$, corresponding to $\bar{C}(\tilde{\mathfrak{c}})$, is flat if and only if $\tilde{\mathfrak{c}}$ is a Lie subalgebra. 
Remark 7.7. Condition (iii) above is equivalent to

(iii') $A d_{G}(t)[\tilde{\mathfrak{c}}] \subset \tilde{\mathfrak{c}}$ for $t \in \bar{H}$, and also to

$\left(\right.$ iii" $\left.^{\prime \prime}\right)[\overline{\mathfrak{h}}, \tilde{\mathfrak{c}}] \subset \tilde{\mathfrak{c}}$ (by the connectedness of $\bar{H}$, see [G-H-V; Vol.II]).

Remark 7.8. A vector subspace $\tilde{\mathfrak{c}} \subset \mathfrak{g}$ that satisfies (i) and (ii) from 7.6 and is a Lie subalgebra of $\mathfrak{g}$ fulfils condition (iii), as well. In the case $\mathfrak{f}=\mathfrak{g}$, i.e. if $\tilde{\mathfrak{c}}+\overline{\mathfrak{h}}=\mathfrak{g}$, we obtain the examples of the $\mathcal{F}$-connections considered in [K3][Ex.7.4.7].

Now, we answer the question:

- When does a connection in $A(G ; H)$ determined by a Lie subalgebra $\tilde{\mathfrak{c}} \subset \mathfrak{g}$ admit a basic adapted one?

Theorem 7.9. If $\tilde{\mathfrak{c}}$ and $\mathfrak{f}$ are Lie subalgebras of $\mathfrak{g}$ such that

(i) $\tilde{\mathfrak{c}} \cap \overline{\mathfrak{h}}=\mathfrak{h}$,

(ii) $\tilde{\mathfrak{c}}+\overline{\mathfrak{h}}=\mathfrak{f}$,

(iii) $\mathfrak{f}$ is reductive in $\mathfrak{g}$,

then the flat connection in $A(G ; H)$ determined by the $\mathcal{F}$-connection $\bar{C}(\tilde{\mathfrak{c}})$ admits a basic adapted one.

[Condition (iii) above holds, for example, in the situation when the connected Lie subgroup of $G$ having $\mathfrak{f}$ as its Lie algebra is compact .]

Proof. The reductivity of $\mathfrak{f}$ in $\mathfrak{g}$ means that the adjoint representation $\operatorname{ad}_{\mathfrak{g}}$ restricted to $\mathfrak{f}, \operatorname{ad}_{\mathfrak{g}} \mid \mathfrak{f}: \mathfrak{f} \rightarrow$ End $\mathfrak{g}$, is semisimple. Therefore there exists an $\left(\operatorname{ad}_{\mathfrak{g}} \mid \mathfrak{f}\right)$ stable vector subspace $\mathfrak{c}_{1} \subset \mathfrak{g}$ complementary to $\mathfrak{f}$, i.e. such that (a) $\left[\mathfrak{f}, \mathfrak{c}_{1}\right] \subset \mathfrak{c}_{1}$, (b) $\mathfrak{c}_{1} \oplus \mathfrak{f}=\mathfrak{g}$. Define

We prove that:

$$
\mathfrak{c}:=\mathfrak{c}_{1} \oplus \tilde{\mathfrak{c}}
$$

$\left(1^{\circ}\right)$ The $G$-left-invariant distribution $\bar{C}(\mathfrak{c})$ on $G$ determined by $\mathfrak{c}$ is an $\mathcal{F}$-connection.

$\left(2^{\circ}\right)$ The connection in $A(G ; H)$ determined by $\bar{C}(\mathfrak{c})$ is basic and adapted to the flat connection determined by $\bar{C}(\tilde{\mathfrak{c}})$.

According to $7.6-7.7$, to prove $\left(1^{\circ}\right)$, we need to observe that (i) $\mathfrak{c} \cap \overline{\mathfrak{h}}=\mathfrak{h}$, (ii) $\mathfrak{c}+\overline{\mathfrak{h}}=\mathfrak{g}$, (iii) $[\overline{\mathfrak{h}}, \mathfrak{c}] \subset \mathfrak{c}$.

(i): Let $v \in \mathfrak{c} \cap \overline{\mathfrak{h}}$. Write $v=v_{1}+\tilde{v}, v_{1} \in \mathfrak{c}_{1}, \tilde{v} \in \tilde{\mathfrak{c}}$. This implies that $v_{1}=v-\tilde{v} \in \mathfrak{c}_{1} \cap(\overline{\mathfrak{h}}+\tilde{\mathfrak{c}})=\mathfrak{c}_{1} \cap \mathfrak{f}=0$. So, $v=\tilde{v} \in \overline{\mathfrak{h}} \cap \tilde{\mathfrak{c}}=\mathfrak{h}$.

(ii): $\mathfrak{c}+\overline{\mathfrak{h}}=\left(\mathfrak{c}_{1} \oplus \tilde{\mathfrak{c}}\right)+\overline{\mathfrak{h}} \subset \mathfrak{c}_{1}+(\tilde{\mathfrak{c}}+\overline{\mathfrak{h}})=\mathfrak{c}_{1}+\mathfrak{f}=\mathfrak{g}$,

(iii): $[\overline{\mathfrak{h}}, \mathfrak{c}]=\left[\overline{\mathfrak{h}}, \mathfrak{c}_{1} \oplus \tilde{\mathfrak{c}}\right] \subset\left[\overline{\mathfrak{h}}, \mathfrak{c}_{1}\right]+[\overline{\mathfrak{h}}, \tilde{\mathfrak{c}}] \subset\left[\mathfrak{f}, \mathfrak{c}_{1}\right]+\tilde{\mathfrak{c}}=\mathfrak{c}_{1}+\tilde{\mathfrak{c}}=\mathfrak{c}$.

Clearly, the connection in $A(G ; H)$ determined by $\mathfrak{c}$ is adapted to that determined by $\tilde{\mathfrak{c}}$. It remains to show that it is also basic. For the purpose, take the curvature form $\bar{\Omega} \in \Omega^{2}\left(G ; Q^{\prime}\right)$ of $\bar{C}(\mathfrak{c})$ (see the proof of Proposition 6.2 for its definition). It is trivial to assert that the connection in $A(G ; H)$ determined by $\mathfrak{c}$ is basic if and only if $i_{v} \bar{\Omega}=0$ for $v \in \bar{F}(\mathfrak{f})$. We prove that this condition can be reduced to

$$
i_{v}\left(\bar{\Omega}_{\mid e}\right)=0 \text { for } v \in \mathfrak{f},
$$

concerning the value of $\bar{\Omega}$ at the unit $e \in G$, due to the commutativity of the diagram

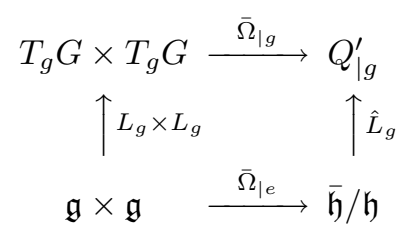


where $L_{g}$ and $\hat{L}_{g}$ are determined (in an evident manner) by the left translations on $G$. We now prove the commutativity of (7.2).

Let $K: T G \rightarrow T G$ be the horizontal projection with respect to the decomposition $T G=E_{b} \oplus \bar{C}\left(\mathfrak{c}_{\mathfrak{u}} \oplus \mathfrak{c}_{1}\right)$ where $\mathfrak{c}_{\mathfrak{u}} \subset \tilde{\mathfrak{c}}$ is any vector subspace such that $\mathfrak{f}=\overline{\mathfrak{h}} \oplus \mathfrak{c}_{\mathfrak{u}}$, and $\bar{C}\left(\mathfrak{c}_{\mathfrak{u}} \oplus \mathfrak{c}_{1}\right)$ is the $G$-left-invariant distribution generated by $\mathfrak{c}_{\mathfrak{u}} \oplus \mathfrak{c}_{1}$. Then we have:

(a) the equality $K \circ X_{v}=X_{K_{\mid e}(v)}$ for $v \in \mathfrak{g}$, where $X_{v}$ stands for the leftinvariant vector field generated by $v$;

(b) for $\bar{\omega} \in \Omega^{1}\left(G ; Q^{\prime}\right)$ - the connection form of $\bar{C}(\mathfrak{c})$, see the proof of $6.2-$ the equality

$$
\bar{\omega}_{\mid g} \circ L_{g}=\hat{L}_{g} \circ \bar{\omega}_{\mid e}
$$

with $\bar{\omega}_{\mid e}$ equal to the composition

$$
\bar{\omega}_{\mid e}: \mathfrak{g} \stackrel{\text { proj }}{\longrightarrow} \mathfrak{g} / \mathfrak{h}=\overline{\mathfrak{h}} / \mathfrak{h} \oplus \mathfrak{c} / \mathfrak{h} \stackrel{\text { proj }}{\longrightarrow} \overline{\mathfrak{h}} / \mathfrak{h} .
$$

From the above and equality (6.2) (which holds for arbitrarily taken vector fields $\left.Y_{1}, Y_{2}\right)$ we obtain, for $v, w \in \mathfrak{g}$,

$$
\begin{aligned}
\bar{\Omega}_{\mid g}\left(L_{g}(v), L_{g}(w)\right) & =\bar{\Omega}\left(X_{v}, X_{w}\right)(g) \\
& =-\bar{\omega}\left(\left[K \circ X_{v}, K \circ X_{w}\right]\right)(g) \\
& =-\bar{\omega}\left(\left[X_{K_{\mid e}(v)}, X_{K_{\mid e}(w)}\right]\right)(g) \\
& =-\bar{\omega}\left(X_{\left.\left[K_{\mid e}(v), K_{\mid e}(w)\right]^{L}\right)(g)}\right. \\
& =-\bar{\omega}_{\mid g}\left(L_{g}\left(\left[K_{\mid e}(v), K_{\mid e}(w)\right]^{L}\right)\right) \\
& \left.=-\hat{L}_{g} \bar{\omega}_{\mid e}\left(\left[K \circ X_{v}, K \circ X_{w}\right]\right)(e)\right) \\
& =\hat{L}_{g} \bar{\Omega}_{\mid e}(v, w)
\end{aligned}
$$

$\left([\cdot, \cdot]^{L}\right.$ denotes the bracket in the left Lie algebra of $\left.G\right)$, which proves the commutativity of diagram (7.2) and, simultaneously, the equality

$$
\bar{\Omega}_{\mid e}(v, w)=-\bar{\omega}_{\mid e}\left(\left[K_{\mid e}(v), K_{\mid e}(w)\right]^{L}\right)=-\bar{\omega}_{\mid e} \circ[\cdot, \cdot]^{L} \circ\left(K_{\mid e} \times K_{\mid e}\right)(v, w) .
$$

This formula and (b) above yield that condition (7.1) is equivalent to $\left[\mathfrak{c}_{\mathfrak{u}}, \mathfrak{c}_{\mathfrak{u}} \oplus \mathfrak{c}_{1}\right] \subset \mathfrak{c}$ because

$$
\bar{\Omega}_{\mid e} \mid \mathfrak{f} \times \mathfrak{g}=0 \quad \Leftrightarrow \quad[\cdot, \cdot]^{L}\left[K_{\mid e}[\mathfrak{f}] \times K_{\mid e}[\mathfrak{g}]\right] \subset \mathfrak{c},
$$

and $K_{\mid e}[\mathfrak{f}]=\mathfrak{c}_{\mathfrak{u}}$ and $\operatorname{Im} K_{\mid e}=\mathfrak{c}_{\mathfrak{u}} \oplus \mathfrak{c}_{1}$.

On the other hand, since $\tilde{\mathfrak{c}}$ is a Lie subalgebra and $\mathfrak{c}_{1}$ is $\left(\operatorname{ad}_{\mathfrak{g}} \mid \mathfrak{f}\right)$-stable, we have

$$
\left[\mathfrak{c}_{\mathfrak{u}}, \mathfrak{c}_{\mathfrak{u}} \oplus \mathfrak{c}_{1}\right] \subset\left[\mathfrak{c}_{\mathfrak{u}}, \mathfrak{c}_{\mathfrak{u}}\right]+\left[\mathfrak{c}_{\mathfrak{u}}, \mathfrak{c}_{1}\right] \subset[\tilde{\mathfrak{c}}, \tilde{\mathfrak{c}}]+\left[\mathfrak{f}, \mathfrak{c}_{1}\right] \subset \tilde{\mathfrak{c}}+\mathfrak{c}_{1}=\mathfrak{c} .
$$

In $[\mathrm{K} 3]$ the following theorem is proved.

Theorem 7.10. [K3, 7.4.2] The Chern-Weil homomorphism $h_{A(G ; H)}$ of the Lie algebroid $A(G ; H)$ makes the diagram

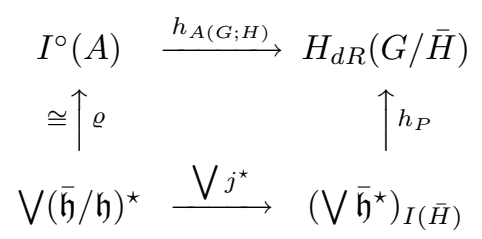

commute for some isomorphism @ of algebras, in which $\bigvee j^{\star}$ is a monomorphism of algebras induced by the canonical projection $j: \overline{\mathfrak{h}} \rightarrow \overline{\mathfrak{h}} / \mathfrak{h}$, whereas $h_{P}$ is the Chern-Weil homomorphism of the $\bar{H}$-principal bundle $P=(P \rightarrow G / \bar{H})$. 
This theorem, Corollary 6.3, Lemma 7.6 and Theorem 7.9 yield the following main theorem of this section:

Theorem 7.11 (Bott's phenomenon for nonclosed Lie subgroups). If the composition

$$
\bigvee^{k}(\overline{\mathfrak{h}} / \mathfrak{h})^{\star} \stackrel{\text { mono }}{\longrightarrow}\left(\bigvee^{k} \overline{\mathfrak{h}}^{\star}\right)_{I(\bar{H})} \stackrel{h_{P}^{2 k}}{\longrightarrow} H_{P}^{2 k}(G / \bar{H})
$$

is nontrivial (for some positive integer $k$ ), then there exists no Lie subalgebra $\mathfrak{c} \subset \mathfrak{g}$ such that

(i) $\mathfrak{c} \cap \overline{\mathfrak{h}}=\mathfrak{h}$,

(ii) $\mathfrak{c}+\overline{\mathfrak{h}}$ is a Lie subalgebra of $\mathfrak{g}$ of codimension $\geq k-1$.

Also, there exists no Lie subalgebra $\mathfrak{c} \subset \mathfrak{g}$ such that

(i) as above,

(ii') $\mathfrak{c}+\overline{\mathfrak{h}}$ is a Lie subalgebra reductive in $\mathfrak{g}$, of codimension $\geq 2 k-1$.

Finally, as an example we consider the case of a compact Lie group and a maximal torus $T \subset G$. For the $T$-principal bundle $P=(G \rightarrow G / T)$, we have [G-H-V, Vol III]

$$
\operatorname{Pont}_{P}^{n-m}=H_{d R}^{n-m}(G / T)(\cong \mathbb{R}),
$$

where $n=\operatorname{dim} G$ and $m=\operatorname{dim} T$. Therefore

$$
h_{P}^{n-m}: \bigvee^{\frac{1}{2}(n-m)} \mathfrak{t}^{\star} \longrightarrow H_{d R}^{n-m}(G / T)
$$

is a nontrivial surjective linear homomorphism ( $\mathfrak{t}$ being the Lie algebra of $T$ ).

Problem 7.12. Find all Lie subalgebras $\mathfrak{h} \subset \mathfrak{t}$ such that the Lie group $H$ determined by $\mathfrak{h}$ is dense in $T$ and the composition

$$
\bigvee^{\frac{1}{2}(n-m)}(\mathfrak{t} / \mathfrak{h})^{\star} \stackrel{\text { mono }}{\longrightarrow} \bigvee^{\frac{1}{2}(n-m)} \mathfrak{t}^{\star} \stackrel{h_{P}^{n-m}}{\longrightarrow} H_{d R}^{n-m}(G / T)
$$

is nontrivial. Any such Lie algebra $\mathfrak{h}$, according to Th.7.11, admits no Lie subalgebra $\mathfrak{c}$ of $\mathfrak{g}$ having the properties for $k=\frac{1}{2}(n-m)$ indicated there. The existence of such a Lie subalgebra $\mathfrak{h}$ was asserted for $G=U(2)$ in [K2, Ex.4.3].

\section{REFERENCES}

[A] A. Andrzejczak, Some characteristic invariants of foliated bundles, Dissertationes Mathematicae, 222, (1984). MR 85h:57034

[A-M] R. Almeida, P.Molino, Suites d'Atiyah et feuilletages transversalement complets, C. R. Acad. Sc. Paris. 300, 1985, Série 1, 13-15. MR 86i:57029

[C-D-W] A Coste, P. Dazord, A. Weinstein, Groupoïdes symplectiques, Publ. Dép. Math. Univ. Lyon Ser. A 2 (1987). MR 90g:58033

[G] W. Greub, Multilinear algebra, Springer-Verlag, New York, 1967. MR 37:222

[G-H-V] W. Greub, S. Halperin, R. Vanstone, Connections, Curvature, and Cohomology. Vols. II, III, Academic Press, New York, 1973, 1976. MR 49:1424, MR 53: 4110

[K-T] F. Kamber, Ph. Tondeur, Foliated Bundles and Characteristic Classes, Lectures Notes in Mathematics 493, Springer-Verlag 1975. MR 53:6587

[K1] J. Kubarski, Lie algebroid of a principal fibre bundle, Publ. Dépt. Math. Univ. Lyons N. S. A 1989, no. 1, 1-66. MR 92h:58206

[K2] $\quad$ A criterion for the minimal closedness of the Lie subalgebra corresponding to a connected nonclosed Lie subgroup, Rev. Mat. Univ. Complut. Madrid 4 (1991), 159-176. MR 93c:22011

[K3] , The Chern-Weil homomorphism of regular Lie algebroids, Publ. Dépt. Math. Univ. Lyons N.S. A (to appear). 
[K4] Tangential Chern-Weil homomorphism, International Symposium/Workshop on Geometric Study of Foliations, November 15-26 1993, Tokyo (to appear)

[L1] P. Libermann, Pseudogroupes infinitésimaux attachés aux pseudogroupes de Lie, Bull. Soc. Math. France, 87, (1959), 409-425. MR 23:A607

[L2] _ Sur les prolongements des fibrés principaux et des groupoïdes différentiables banachiques, Seminaire de mathématiques superieures - été 1969, Analyse Globale, Les Presses de l'Université de Montréal, 1971, pp. 7-108. MR 50:8688

[MA] K. Mackenzie, Lie groupoids and Lie algebroids in differential, Geometry, London Mathematical Society Lecture Note Series 124, Cambridge, 1987. MR 89g:58225

[MO1] P. Molino, Etude des feuilletages transversalement complets et applications, Ann. Sci. Ecole Norm. Sup., (4) 10 (1977), 289-307. MR 56:16649

[MO2] , Riemannian Foliations, Progresss in Mathematics, Vol. 73, Birkhäuser, Boston, Basel, 1988. MR 89b:53054

[P] J. Pradines, Théorie de Lie pour les groupoïdes différentiables, Atti Conv. Intern. Geom. 7 Diff. Bologne, 1967, Bologna-Amsterdam.

[NVQ] Ngo-Van-Que, Du prolongement des espaces fibrés et des structure infinitésimales, Ann. Inst. Fourier, (Grenoble), 17 (1967), 157-223. MR 36:4468

[T1] N. Teleman, Cohomology of Lie algebras, Global Analysis and its Applications, Vol. III, Intern. Course, Trieste 1972, Internat. Atomic Energy Agency, Vienna 1974, pp. 195-202. MR 55:9112

[T2] - A characteristic ring of a Lie algebra extension, Accad. Naz. Lincei. Rend. Cl. Sci. Fis. Mat. Natur. (8), 52 (1972), 498-506 and 708-711, 1972. MR 49:6251

Institute of Mathematics, Technical University of Lodz, PL-90-924 Lodz, Al. PoLITECHNIKI 11, Poland

E-mail address: kubarski@Lodz1.p.lodz.pl 